\title{
The Organization of Visually Mediated Actions in a Subject without Eye Movements
}

\author{
Michael F. Land, Sophie M. Furneaux and lain D. Gilchrist ${ }^{1}$ \\ School of Biological Sciences, University of Sussex, Brighton BN1 9QG and ${ }^{1}$ Department of Experimental Psychology, \\ University of Bristol, 8 Woodlands Road, Bristol BS8 1TN, UK
}

\begin{abstract}
We investigated the visual strategy of a subject without eye movements (Al), comparing her with normal subjects on the 'real-life' task of making a cup of tea. Differences in overall performance were surprisingly few. She took no more time than the controls to complete the tea-making task and the division of the task into object-related actions was essentially similar. However, the way Al took in visual information was very different from the normal subjects who used a typical 'saccade and fixate' strategy when moving between and scrutinizing objects. Al made saccades with the head, which were on average 1.5 times larger than the eye-head saccades of the controls and lasted four times as long, meaning that Al would have had impaired vision for more of the time than the controls. She also made only approximately one-third as many saccades as normals during the same task. However, she had another strategy, 'slow drift', in which she allowed her eyes to move smoothly across the scene at speeds of up to $30 \%$ s. Such movements were never seen in the controls, and we assume that Al used them to offset the cost in time of the slow head saccades, even though they had their own cost in terms of reduced resolution. We demonstrate that these differences have a minimal effect on the timings of events during an object-related action. We discuss supervisory checking operations within actions, and consider what information is needed for appropriate gaze control during object-related actions.
\end{abstract}

\section{Introduction}

In a previous paper (Land et al., 1999) we examined the relationships between eye movements and actions during the semi-automatic task of tea making. We showed that eye movements precede the actions they guide by about $0.5 \mathrm{~s}$, and provide the motor system with specific types of information used in the formulation and control of the component actions of the task. Normal subjects obtain this information using an eye movement strategy that involves an alternation between saccades, where the eyes are moving rapidly, and fixations, where the gaze is held still in space (Yarbus, 1967; Carpenter, 1988). Vision is largely suppressed during saccades, and information from the world can normally only be acquired during fixations, so this typical visual strategy involves a trade-off between keeping the eyes still and bringing new parts of the visual field on to the fovea. In this paper we report a subject who has no eye movements, and employs a quite different strategy to shift her gaze during execution of the task. We ask whether this altered strategy affects the way that information is obtained and, hence, the way the task itself is executed.

Our subject (AI) has total ophthalmoplegia (eye paralysis) resulting from extra-ocular muscle fibrosis. This means that her eyes are unable to move relative to her head, making the normal 'eye saccade and fixate' visual strategy impossible; in order to change her position of fixation, she must move her head and/or body. Gilchrist et al. (1998) provide several lines of evidence showing that she is replacing eye saccades with head movements. Qualitatively, these head movements seem similar to saccades, and the evidence suggests that AI uses the normal eye saccade control system to control her head movements. A similar conclusion was reached by Gaymard et al. (2000) in a study of a patient with acute acquired ophthalmoplegia. However, because of the extra inertia of the head, AI's head saccades take about four times longer than normal eye-head saccades: about $400 \mathrm{~ms}$ for a $30^{\circ}$ gaze shift compared with $100 \mathrm{~ms}$. This difference in duration means that to view the same range of task-related objects in the same time as normal subjects she must adopt a different strategy. Our main finding was that AI drastically reduces the number of saccades she makes, to one-third that of normals. However, to allow for small shifts in gaze she lets her gaze 'drift' at speeds of up to $30 \%$, a type of movement never seen in normal controls.

AI does not report any major visual problems associated 
with her deficit, and a previous study by Gilchrist et al. (1998) showed that her reading ability and speed are well within standard limits, although her silent reading is towards the slower end of normal. In this study we were concerned with the effects that her unusual eye movement strategy might have on the execution of the tea-making task. In particular, the reduced number of saccades might be expected to change the timings of the eye movements relative to the limb movements they relate to. In fact we found that there were no differences in the types of information supplied by the eyes to the limbs, or in the way the task was executed. This implies that the basic structure of the task, involving a succession of object-related acts (ORA; Land et al., 1999) or 'A1 units of action' (Schwartz et al., 1991, 1995), is robust, and essentially independent of the eye movement strategy used to acquire the relevant information. The visual prerequisites for this type of serial behaviour are considered in the Discussion.

\section{Materials and methods}

\section{The subject}

AI, a right-handed female, was, at the time of testing, a 22year-old university undergraduate reading English. Previous reports on her visual behaviour can be found in Gilchrist et al. (1997, 1998). Her medical records show that she was diagnosed as having congenital oculofibrosis of unknown aetiology, present since birth; she has never had more than rudimentary eye movements. She shows no optokinetic response (eye rotation to a stripe pattern) and the largest eye movements that have previously been recorded were $\pm 0.5^{\circ}$ (Gilchrist et al., 1997). AI has normal pupillary and blink reflexes. On the Ishihara colour vision tests she appears to have normal colour vision. Stereo vision is absent, and the left eye has a divergent squint. She shows strong suppression of the left eye, although we have unequivocal evidence from our recordings that this eye is used occasionally to fixate objects. Visual acuity while wearing corrective lenses, using the Snellen letter chart, is $6 / 12$ in both left and right eyes. A more extensive history can be found in Gilchrist et al. (1998). AI gave her informed consent for these studies.

To act as controls, three participants with normal eye movements were used. The recordings of the tea-making task were taken from the subjects used by Land et al. (1999).

\section{Methods}

The direction of fixation for all subjects was recorded using a head-mounted video camera system (Land, 1993; Land et al., 1999). This is a non-intrusive device that allows normal head and body movement during tracking, and was used to monitor AI's head movements, and the eye and head movements of the controls. The device produces a splitscreen video recording using two mirrors: a part-silvered mirror images the scene ahead and a concave mirror images the subject's left eye. The subjects were precisely calibrated at the beginning and the end of each recording session to ensure that no slippage of the headset had occurred.

The position of gaze in space was measured off-line from the videos, at a time resolution of $50 \mathrm{~Hz}$. This was carried out using a computer model of the eye fitted to the outline of the iris, using its shape and position. From this, gaze direction was determined to an accuracy of approximately $1^{\circ}$ in the normal subjects. Using these coordinates a secondgeneration video was produced which showed the direction of gaze superimposed as a dot on to the scene ahead. In AI's case this procedure directly determined head (and gaze) direction. For the control subjects, head movement was retrieved from the movement of objects in the scene video and added to the eye movement record to give gaze direction.

The subjects were required to make a cup of tea in a small rectangular kitchen. The room had a worktop (counter) to the left of the door, a sink directly in front and another worktop and refrigerators on the right [see Land et al. (1999) for more details]. The subjects wore the eye tracker and were also filmed from an external viewpoint so that information about their hand and body movements in space could be compared with their eye movements. The normal subjects and their visual strategies when making tea have been described in detail previously (Land et al., 1999).

\section{Results \\ Eye movement strategies}

AI made a cup of tea following much the same sequence of actions as described in Land et al. (1999). She completed the task without fault in 4 min $50 \mathrm{~s}$, which was similar to the three control subjects $($ mean $=4 \min 46 \mathrm{~s}$, range $=$ 3 min 2 s to 5 min 47 s) In Figs 1 and 2 her gaze movement strategy is compared with a normal control (ML) for the very first part of the task-filling the kettle. Both AI and ML first examined the kettle, then lifted it and took it to the sink whilst removing the lid in transit. They then turned on the right-hand tap and put the kettle beneath it, whilst fixating on the water stream. ML (Fig. 1a) made a total of 26 fixations brought about by combined eye and head saccades. The other two control subjects both made 25 fixations during the same series of actions. AI (Fig. 1b) made 10 head saccades between 11 fixation points. Over the whole task we found that AI made far fewer saccades than the controls, by a factor of three (152 compared with the control mean of 453 , range $=$ 401-545). The impression one has is of sensible economy; AI used only one fixation (on the taps, for example) where a normal subject would use three. The interesting question seems not to be why AI uses so few saccades, but why normals use so many.

Figure 2 shows records of parts of the same two sequences. In Fig. 2a ML is examining the kettle for the first $4 \mathrm{~s}$ making a total of 12 small saccades (arrowed). The gaze trace shows almost stationary fixations between the saccades. The 

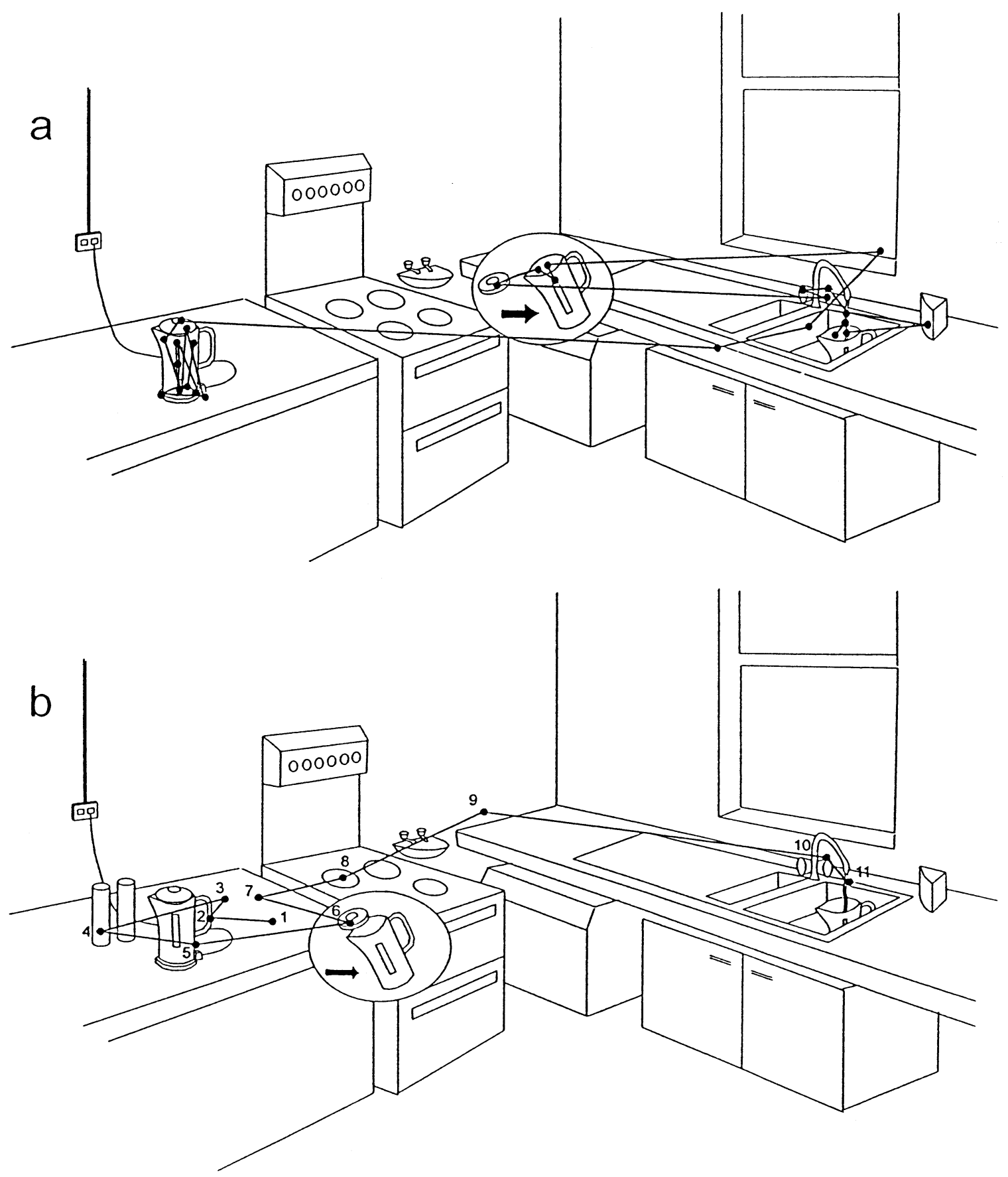

Fig. 1. (a) Fixations made by a normal subject during the first section of a tea-making task (filling the kettle). (b) Fixations made by AI during the same episode. The objects looked at were very similar in the two cases, but AI used less than half as many fixations. Her fixations were not stationary, as can be seen in Fig. 2b.

saccades are clearly visible on the eye trace because they are the result of eye muscle activity. However, the eye record also shows a large $\left(30^{\circ}\right)$ leftward sweep between 0.5 and $2.0 \mathrm{~s}$, which does not appear on the gaze record. This movement is equal and opposite to a rightward movement of the head and is a typical compensatory response brought about by the vestibulo-ocular reflex which automatically moves the eyes in the opposite direction to head rotation. The effect on gaze is that head and eye movements cancel, leaving stationary fixations. After viewing the kettle, gaze moves off to the sink on the right (see Fig. 1a) with three combined head and eye saccades, and in this case the fixations between the saccades are not fully stabilized.

In AI's record (Fig. 2b) the head and gaze records are one and the same, and there is no eye record. The upper record shows eye velocity and in particular it shows 'spikes' of between 50 and $100 \%$ s, corresponding to the head saccades in the position record. Numbers correspond to the fixations 

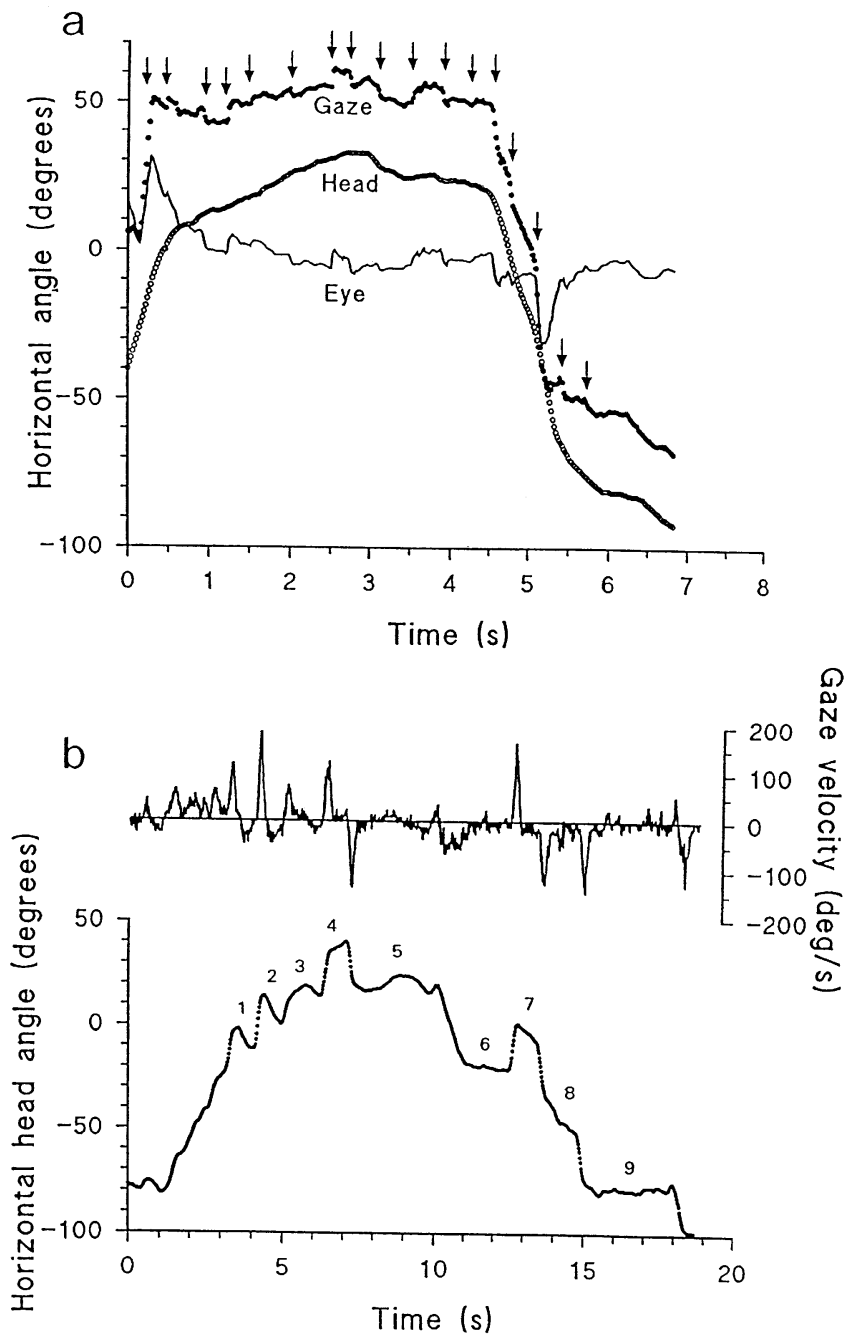

Fig. 2. Records of gaze of a normal subject (a) and AI (b) during the early part of the episode illustrated in Fig. 1. (a) shows records of eye-in-head and head-in-space directions, and gaze direction, which is the sum of the two. Saccades are arrowed. In (b) there is only one record because AI has no eye movements, so gaze and head-in-space records are the same. AI showed distinct head saccades, indicated by 'spikes' in the velocity record. However, during 'fixations' her gaze drifted at speeds of up to $30^{\circ} \%$. The numbers in (b) correspond to the locations shown in Fig. 1b. Note that the sequence in (b) takes in more acts than (a), hence the difference in time scale.

in Fig. 1b. Two features are evident. First, the head angle trace does contain a number of fast head saccades which shift gaze by $20-30^{\circ}$. These are not seen in the head movement record of ML and we assume that they have the same gaze shifting function as the eye saccades in Fig. 2a. Second, during the 'fixation' periods between saccades, the head and, hence, gaze are far from stationary. The rate of drift varies from $0 \%$ (fixation 9) to about $30 \%$ s (fixations 1 and 2). The long movement between fixations 5 and 6 is probably a drift rather than a saccade. The velocity of $42^{\circ} \%$ is high compared with the other drifts, but for a head saccade of this size $\left(40^{\circ}\right)$ a peak velocity of close to $200^{\circ}$ /s would be expected (Zangemeister et al., 1981).

Statistical details of AI's head saccades will be given elsewhere, but three features are important for this paper. First, they were larger than normals (mean $32.8^{\circ}$; control mean $19.5^{\circ}$; range $18.1-20.2^{\circ}: P<0.001$ for difference of means: $t$-test). This is probably because many of the seemingly supernumary small saccades made by the normal subjects were omitted, but the large gaze shifts from one object to the next were not. Second, they took much longer to execute. A $30^{\circ}$ head saccade made by AI took $0.4 \mathrm{~s}$ to complete compared with just under $0.1 \mathrm{~s}$ for a typical eye saccade of the same size (Carpenter, 1988, p. 71). An obvious consequence of this is that it would be impossible for AI to make three saccades a second, as normals do, because there would be no time at all for fixation and, hence, clear vision. Third, AI's head saccades were slow: a $30^{\circ}$ gaze shift had a maximum velocity of $150 \%$ s compared with close to $800^{\circ} / \mathrm{s}$ for a $30^{\circ}$ eye saccade (Carpenter, 1988, p. 72). Because it takes a long time to accelerate and decelerate the head, compared with the eye, the average velocity of a head saccade is only about half the maximum, $75 \%$ in this case. This is slow enough for there to be a question about whether AI is as blind during a head saccade as normals are during an eye saccade. Although AI does show saccadic suppression (Gilchrist et al., 1998), this peaks around the beginning of the movement and by $50 \mathrm{~ms}$ into the movement the detectability of a flash is only lowered by about $20 \%$. Thus, certainly towards the end of a head saccade, AI is likely to have some degree of useful vision.

\section{Execution of the task}

As Fig. 1 shows, AI performed the same sequence of actions in filling the kettle as the control ML. The only difference was that she used fewer fixations in the process. This was true for the whole task; AI's actions matched those of the controls. (There was a considerable degree of variability amongst the controls. Not all actions-when to find the mug, for example-needed to be performed in an exact sequence.)

We investigated the timings of gaze changes in relation to the component acts of the task, thinking that these were likely to be affected by the much longer duration of the head saccades of AI. Tasks like tea making can be divided into subtasks, the most basic of which is an ORA (Land et al., 1999). This is very similar to the 'A1 unit of action' of Schwartz et al. (1991, 1995), which they define as 'simple actions that transform the state or place of an entity through manual manipulation'. Our definition of an ORA differs only in that it embraces the eye movements that accompany the action. Like A1 actions, ORAs do not provide a perfect description, because actions may be nested within each other (for example, in Fig. 1 the lid is removed from the teapot while the latter is being taken to the sink) and occasionally the two hands are engaged in unrelated acts. Nevertheless, ORAs provide the least ambiguous way of dividing up an action sequence. Their other advantage is that they nearly always begin with a 'defining moment' when gaze moves from one object to the next, and this can be used as a sharp 
A.I.

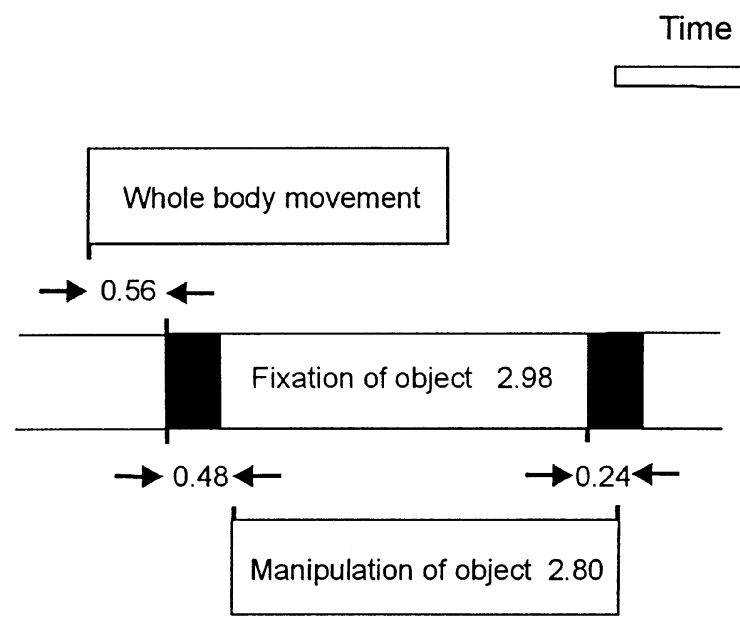

Controls

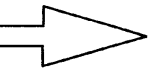

1 second

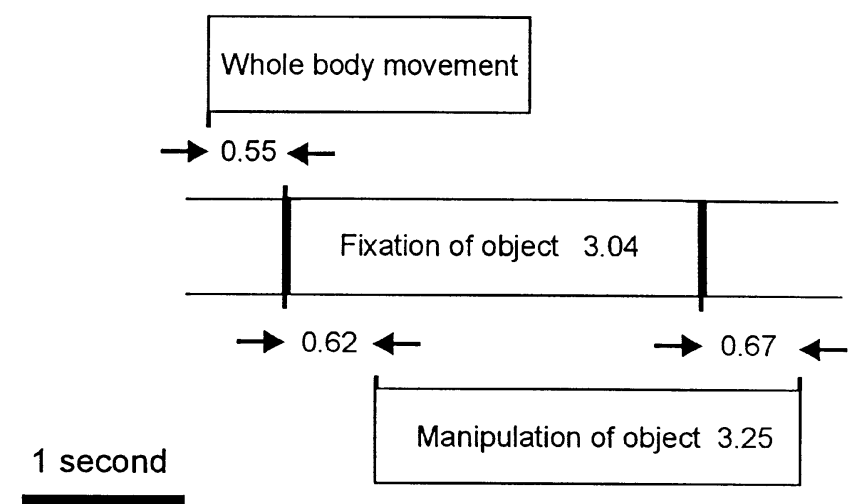

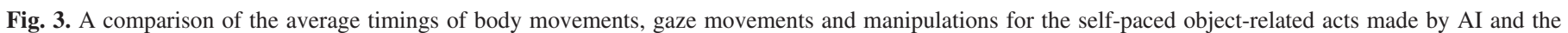

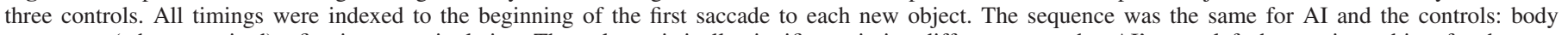

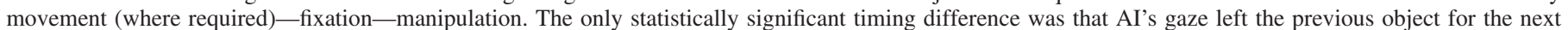

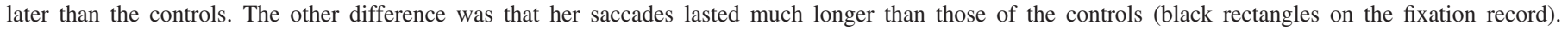
Numbers are times in seconds. For further details see Land et al. (1999).

temporal marker to establish the beginning and end of each action.

Figure 3 in Land et al. (1999) gives details of all 43 ORAs for a single tea-making sequence. For the two other control subjects, and AI, the pattern was very similar. The numbers of ORAs were 41 and 36 for the other two controls and 48 for AI. The differences are mainly accounted for by the extent to which objects were picked up and set down for no good reason. The minimum number of ORAs needed for the task is probably about 30 . Figure 3 shows the timing of the components of all ORAs, for the mean of the three controls and for AI. All timings were indexed to the beginning of the saccade (head, eye or combination) which found the object to be dealt with in the subsequent ORA. For both the controls and AI the patterns were very similar. If a body movement were required (for example, because the mugs were on the opposite side of the kitchen from the teapot), then that movement begins about $0.5 \mathrm{~s}$ before the first saccade to the relevant object. This saccade typically led the first signs of a movement to contact the object, again by about $0.5 \mathrm{~s}$. Selfpaced actions - those that do not require an enforced delay, such as waiting for the kettle to boil-usually lasted about 3 s. At the end of each ORA gaze moved on to the object of the next action, generally before the previous manipulation was complete. The only statistically significant difference ( $t$-test, $P<0.05$ ) between AI and the controls was this anticipatory gaze movement. AI moved later: $0.24 \mathrm{~s}$ before completion rather than $0.67 \mathrm{~s}$. No obvious reason for this, related to her condition, comes to mind. Apart from this, AI's visual dealings with objects she interacted with were barely different from those of the normal controls.

In Land et al. (1999) we proposed that particular fixations had one of four different functions: locating objects possibly to be used later in the process; directing the hand or object in the hand to a new location; guiding the approach of one object to another (e.g. lid to kettle); checking the state of some variable (e.g. water level) or the completion of a subtask. Hayhoe (2000) found that the same analysis fitted equally well in another culinary task-making a peanut butter and jelly sandwich. It was particularly easy to classify AI's fixations according to this scheme, because for the majority their function was clear (in the sense that the beginning of the fixation immediately preceded a particular action). On that basis, $11 \%$ of the 152 fixations were locating fixations involved in episodes of search, $38 \%$ were concerned with directing the hands, $2 \%$ with guiding and $11 \%$ with checking. There were two other categories: brief fixations made during turning and walking, which were probably nothing more than punctuations in very large gaze shifts $(20 \%)$ and fixations to which no function could be ascribed (18\%). The last category included episodes where AI was looking out of the window while the kettle came to the boil. The main difference between AI and the controls was that in the latter the number of fixations in the 'no obvious function' category was much larger (60-70\%). In other respects the distributions were similar.

On three occasions, each lasting a few seconds, we found that AI was using her non-dominant left eye. This manifested itself because she suddenly directed her hand to an object which was about $30^{\circ}$ away from the part of the field she used to target everything else. In the controls the eyes were never more than a few degrees from the target during directing fixations, so we assume that AI must have been using her other eye. On the three occasions this happened the location in the field of view was the same, about $30^{\circ}$ from her usual direction of regard. 


\section{Discussion}

\section{How many fixations are needed to control an action?}

The most striking feature of AI's performance was that the number of fixations she made was only one-third of the number made by the controls. In Land et al. (1999) we commented that a clear function could be attributed to only about one-third of the fixations made by the three subjects, the remainder seeming less necessary for the control of actions. With AI's reduced fixation number the proportion of fixations with an attributable function was much higher $(74 \%$, if the brief fixations made during large gaze shifts are ignored). Because AI's overall performance, in both dexterity and speed, was unimpaired, it would seem on the face of it that two-thirds of the control subjects' fixations were unnecessary. This is probably not really true. Both AI and the controls used large saccades (head or eye plus head) to get from one object to the next in the action sequence. An analysis of all saccades made by the controls (Land and Hayhoe, 2001) showed that between-object saccades had a mean size of $47^{\circ}$, even larger than AI's saccades (mean $32.8^{\circ}$ ), whereas within-object saccades had a mean size of only $8^{\circ}$. Clearly the latter are in the nature of minor adjustments. AI also made adjustments, but she used slow drifts to move short distances around objects, presumably because of the time penalty associated with head saccades. Why might such minor adjustments be necessary? The most plausible reason is that more resolution is required for some aspects of the task, and that means bringing the fovea closer to the relevant part of the object under scrutiny. For AI such a strategy is two-edged, as letting the eyes drift at speeds of $10-30 \%$ will substantially reduce the resolution of fine detail (Carpenter, 1991). How much extra information is obtained from the use of either strategy-saccade or driftremains to be explored.

\section{Unconscious supervision}

In this study and its predecessor (Land et al., 1999) we have found that the eyes are frequently engaged in checking or supervisory operations. For example, making sure that the kettle is held under the water stream from the tap, checking the water level as the kettle fills, ensuring that the tea is poured into the cup. Other operations, such as guiding a lid on to the teapot, also have a supervisory quality to them.

These observations seem to raise problems for schemes, such as that of Norman and Shallice (1986), where automated behaviour is controlled by schemas which are 'routine programs for the control of overlearned skills', but non-routine activities, and intervention when there are problems with automated activities, are dealt with by the 'supervisory attention system'. This system is associated with the frontal lobes of the cortex and involves conscious action. What we see in tea making, and no doubt other similar activities, is supervision being exerted during what are at best semi-automated activities (in the sense that we often perform them while our attention is taken up by the news on the radio). This is not really a surprising finding. Some eye movement functions are essentially open loop (directing the hand typically requires only one glance to establish an object's position and gaze has usually moved on before contact is made), but others require vision to close the feedback loop (in filling a kettle the eyes are needed to detect when the water has reached a criterion level). We argue that in many automated activities that involve visual feedback loops, a degree of supervision is essential. However, this does not imply the same degree of top-down intervention implied by the activation of the 'supervisory attention system', and indeed is largely unconscious. Nevertheless, what we have shown is that there is an element of supervision and attentiveness even at the lowest level of the schema system.

\section{What does the eye movement system need to know?}

We have seen that gaze movements (whether performed by the eyes or, in AI's case, the head) normally precede the first movements of the hands in an ORA (Fig. 3). The gaze control system is thus activated before the beginning of the action itself. It is not just called up when some visual information is needed; it is a planned part of the whole process. Our question here is "What information does the gaze control system itself need in order to get the eyes into a position to be of use to the control system for object manipulation?'

As we have seen here and elsewhere, the most important function of gaze control is to shift the fovea from one object to the next in the 'script', as each ORA is completed. This means that the system must know which object to select and where it is. This is far from simple. The object must be recognized somewhere in the visual periphery, which means that an appropriate search image must be activated across large parts of the visual field. Positional information is needed, and this can come from two sources: memory and immediate vision. In many instances a subject would make an appropriate turn to the location of an object which is out of sight on the other side of the room, and it is a good assumption that the object's position was established in an earlier phase of the task in a 'locating' fixation. There were a number of instances among the control subjects where an object was fixated a minute or more prior to its ultimate use, and when it was time to fetch the object a 'memory' saccade took gaze to within $20^{\circ}$. This was then followed by one or two visually guided saccades which resulted in fixation (Land et al., 1999). Object acquisition thus involves a complex interplay between object recognition, spatial memory and vision.

When manipulation begins, the motor system of the arms and hands needs appropriate instructions about the action they must take. Vision may have a simple directing role here, or in the case of 'guiding' it may have a more active one, 
OBJECT RELATED ACT

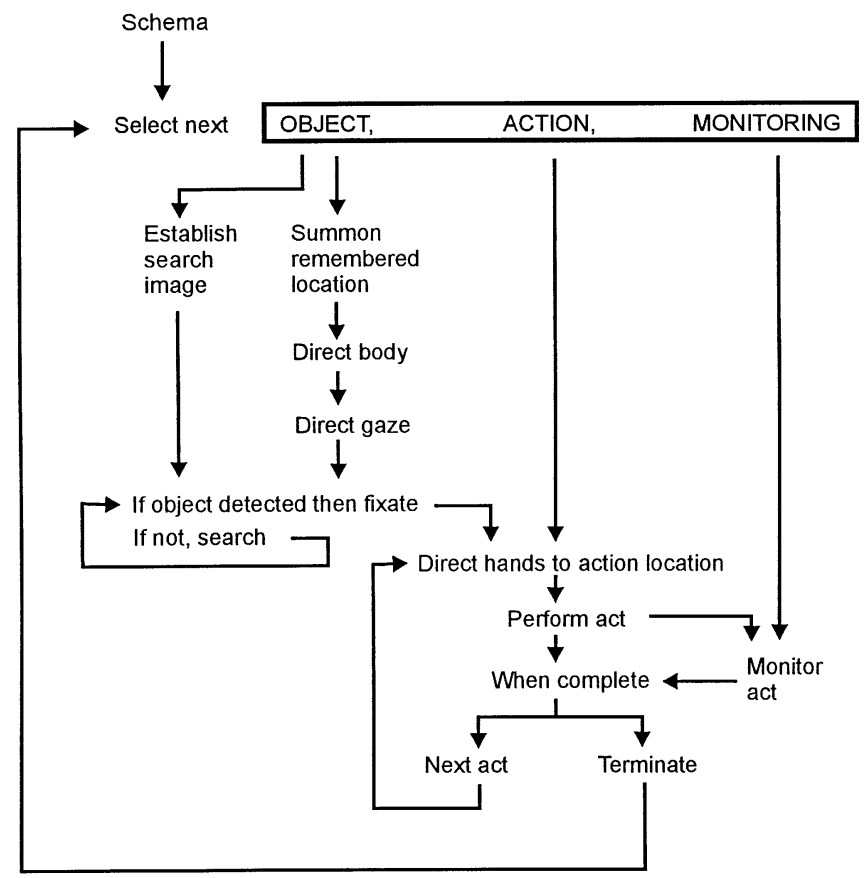

Fig. 4. Scheme illustrating the flow of information during an object-related act. At the beginning of each object-related act the schema (or script) must supply the visual system with information about the identity of the next object, the oculomotor system with information about its location, and the motor system about the action(s) to be performed. The visual and oculomotor systems also require information about the monitoring actions to be performed. The action commences once the object is located, and terminates when the monitoring indicates that the appropriate condition is fulfilled. Further actions on the same object may be initiated or the action terminated and the script consulted again for the instructions for the next action.

with gaze alternating between two objects as they approach each other. In addition, there are checking operations, and the eyes need to know where and what to check. This too must be part of the instructions that the gaze control system receives, presumably at the initiation of each new ORA.

Figure 4 is a summary of the information flow during a single ORA. On (or before) the end of the preceding action, the central executive (supposedly in the frontal lobes) supplies the gaze control system and the motor control systems of the limbs with information about the object (identity and location), the manipulations to be performed and the checking operations required. First the eyes locate the object using memory and direct vision or, if that fails, by a search routine. When the object is located manipulation begins, watched over by the eyes which check to see that each operation is properly completed. When each subact is complete a further manipulation of the same object may follow (e.g. take down the sweeteners, add one to the cup, replace on the shelf), or if there is no further subact on the same object the action as a whole is terminated, the present set of instructions is abandoned and those for the next ORA are sought.

Even though we are discussing here the most basic level in the schema system of Norman and Shallice (1986), it is clear that the complexity is considerable and irreducible. Furthermore, much of the brain is likely to be involved. On current understanding, the frontal lobes would be responsible for the instruction sequence, the parietal lobes for the motor execution of both eye and limb movements, the temporal lobes for object identification and possibly the hippocampus for spatial location.

\section{Acknowledgement}

This study was supported in part by a grant to MFL from the Gatsby Foundation.

\section{References}

Carpenter RHS. Movements of the eyes, 2nd edn. London: Pion, 1988.

Carpenter RHS. The visual origins of ocular motility. In: Carpenter RHS, ed. Vision and visual dysfunction, Vol. 8. Basingstoke, UK: Macmillan, 1991, pp. $1-10$.

Gaymard B, Siegler I, Rivaud-Pechoux S, Israel I, Pierrot-Deseilligny C, Berthoz A. A common mechanism for the control of eye and head movements in humans. Annals of Neurology 2000; 47: 819-22.

Gilchrist ID, Brown V, Findlay JM. Saccades without eye movements. Nature 1997; 390: 130-1.

Gilchrist ID, Brown V, Findlay JM, Clarke MP. Using the eye-movement system to control the head. Proceedings of the Royal Society of London B 1998; 265: 1831-6.

Hayhoe M. Vision using routines: a functional account of vision. Visual Cognition 2000; 7: 43-64.

Land MF. Eye-head co-ordination during driving. Proceedings of the IEEE Systems, Man and Cybernetics Conference, Le Touquet 1993; 3: 490-4.

Land MF, Hayhoe M. In what ways do eye movements contribute to everyday activities? Vision Research 2001; 41: 3559-65.

Land MF, Mennie N, Rusted J. The roles of vision and eye movements in the control of activities of daily living. Perception 1999; 28: 1311-28.

Norman DA, Shallice T. Attention to action: willed and automatic control of behaviour. In: Davidson GE, Schwartz GE, Shapiro D, editors. Consciousness and self-regulation: advances in research and theory, Vol. 4 New York: Plenum, 1986: 1-8.

Schwartz MF, Reed ES, Montgomery MW, Palmer C, Mayer NH. The quantitative description of action disorganisation after brain damage: a case study. Cognitive Neuropsychology 1991; 8: 381-414.

Schwartz MF, Montgomery MW, Fitzpatrick-DeSalme EJ, Ochipa C, Coslett HB, Mayer NH. Analysis of a disorder of everyday action. Cognitive Neuropsychology 1995; 12: 863-92.

Yarbus A. Eye movements and vision. New York: Plenum, 1967.

Zangemeister WH, Jones A, Stark L. Dynamics of head movement trajectories: main sequence relationship. Experimental Neurology 1981; 71: 76-91. 


\title{
The organization of visually mediated actions in a subject without eye movements
}

\section{F. Land, S. M. Furneaux and I. D. Gilchrist}

\begin{abstract}
We investigated the visual strategy of a subject without eye movements (AI), comparing her with normal subjects on the 'real-life' task of making a cup of tea. Differences in overall performance were surprisingly few. She took no more time than the controls to complete the tea-making task and the division of the task into object-related actions was essentially similar. However, the way AI took in visual information was very different from the normal subjects who used a typical 'saccade and fixate' strategy when moving between and scrutinizing objects. AI made saccades with the head, which were on average 1.5 times larger than the eye-head saccades of the controls and lasted four times as long, meaning that AI would have had impaired vision for more of the time than the controls. She also made only approximately one-third as many saccades as normals during the same task. However, she had another strategy, 'slow drift', in which she allowed her eyes to move smoothly across the scene at speeds of up to $30 \%$ s. Such movements were never seen in the controls, and we assume that AI used them to offset the cost in time of the slow head saccades, even though they had their own cost in terms of reduced resolution. We demonstrate that these differences have a minimal effect on the timings of events during an object-related action. We discuss supervisory checking operations within actions, and consider what information is needed for appropriate gaze control during object-related actions.
\end{abstract}

\section{Journal}

Neurocase 2002; 8: 80-87

Neurocase Reference Number:

$\mathrm{O} 246$

Primary diagnosis of interest

Ophthalmoplegia resulting from fibrosis of the eye muscles

\section{Author's designation of case}

AI

\section{Key theoretical issue}

- Role of gaze movements in directing action

Key words: eye movement; vision; control of action; schema; supervisory control

\section{Standardized assessment}

Comparison with three normal subjects on the same task (making tea)

\section{Language}

English 